\title{
An improved prediction method of water consumption rate considering aftereffects for short-term dispatching of hydropower station
}

\author{
Xiao Chen ${ }^{1,2, *}$, Jianzhong Zhou ${ }^{1,2, *}$, Benjun $\mathrm{Jia}^{1,2}$, Yuqi Yang ${ }^{1,2}$, and $\mathrm{Li} \mathrm{Li}^{1,2}$ \\ ${ }^{1}$ School of Hydropower and Information Engineering, Huazhong University of Science and Technology, Wuhan, 430074, P R China \\ ${ }^{2}$ Hubei Key Laboratory of Digital Valley Science and Technology, Wuhan 430074, China
}

\begin{abstract}
Accurate and rapid output calculation of hydropower station (HS) is an important research item in reservoir dispatching neighborhood. There have existed many methods to calculate output in generation scheduling models with different time scale. But for the large HS with multiple units, it is still difficult to calculate output quickly and accurately in short-term generation dispatching. Therefore, in this paper, an improved method of water consumption rate (IWCR) considering aftereffects is proposed. The Three Gorges Hydropower Station (TGHS) in China is selected as the case study, and the prediction water consumption rate (WCR) results are obtained with IWCR and classical water consumption rate method (CWCR). The results show that 1) The mean absolute deviation (MAD) on the left and right bank of TGHS is significantly superior to the $M A D$ calculated by CWCR, and reduce $0.578 \mathrm{~m}^{3} /(\mathrm{s} \cdot \mathrm{wkw})$ and 0.569 $\mathrm{m}^{3} /(\mathrm{s} \cdot \mathrm{wkw})$ respectively. 2) In low relative deviation interval, there are more prediction WCR periods with IWCR. Therefore, the IWCR method can lead to the plan scheme more consistent with actual operation process, and the security of TGHS and Gezhouba is stronger.
\end{abstract}

\section{Introduction}

Rapid and accurate calculation of output is of great significance to the efficient and safe operation of HS [12]. Time scale, calculation precision and timeliness of the scheduling model are very important factors in selecting the way of output calculation. In most of the actual scheduling processes, dispatchers will combine precision and efficiency of output calculation synthetically, making the calculation results available in an acceptable precision range. In the neighborhood of reservoir operation, the existing output calculation methods are mainly as follows: 1) The efficiency coefficient method (ECM) [3-5]; 2) The classical water consumption rate method (CWCR) [6-7]; 3) Refinement calculation methods based on the single unit flow curve (RC) [8-9].

ECM is the most important and commonest method of output calculation in long term generation scheduling. In this method, output is obtained by multiplying the net head, power generation flow and efficiency coefficient directly [3]. The efficiency coefficient can usually be set to a constant value depending on the operation of HS over many years. The outflow and net head of the whole plant can also be obtained by water balance calculation and head calculation formula easily. Therefore, this method is quite efficient. But the disadvantages of ECM are also obvious. The calculation parameters of this method are the mean values of the whole plant. For a hydropower plant with a large number of units, the efficiency of different units often varies greatly. At the same time, the head loss curves of different units are not the same. The net head and efficiency coefficient of the whole plant are often unable to meet the calculation accuracy of short-term power generation dispatching on daily or hourly scale. Therefore, in the vast majority of short-term scheduling, the ECM cannot be used $[1,8]$. Compared with ECM, the calculation accuracy of output by CWCR is generally better. (The flow and water volume can be converted to each other. Therefore, without special instructions, the WCR in this paper refers to the flow consumption rate.) The CWCR method is the same as the ECM method, which also treats the units of whole plant as a whole [6]. It typically takes four steps to perform the calculation. First, the historical operation data of HS, including the power generation flow, output and gross head data of the whole plant needs to be collected. Second, the WCR of the whole plant, which corresponds to gross head, is calculated by the power generation flow divided by output. Third, the experience curve (EC) between gross head and WCR is fitted by historical data. Finally, the WCR is obtained by querying the EC with gross head as the parameter. In the multi-day generation scheduling, the CWCR method is a common method to calculate output.

The RC methods are all based on the single unit flow curve (UFC). The UFC of a single unit, which is threedimensional curve among net head, generation flow and output, can generally be obtained from the unit model experimental data. Simultaneously, with the operation of

* Corresponding author: xiao_ch@hust.edu.cn; jz.zhou@hust.edu.cn 
HS, the station itself can also check and correct UFC. When the net head is already known, the generation flow or output can be calculated with high precision. Therefore, the unit commitment problem based on UFC is a very important research neighborhood in short-term or real-time scheduling [8,9]. However, for the HS with a large number of units and multiple types, traditional solving algorithms, such as dynamic programming (DP) [10], are unable to meet computational timeliness due to the dimension disaster. In recent years, many intelligence algorithms have appeared in unit commitment. However, the biggest disadvantage of these algorithms is that the calculation results are not stable, so these adaptabilities are poor [8].

Seen from above analysis, although there have existed many calculation methods for output, the problem of fast and accurate calculation for short-term and real-time scheduling of large HS is still intractable. Based on this dilemma, in this paper, we propose an improved WCR calculation method for short-term scheduling considering aftereffect.

The remaining chapters of this paper are arranged as follows: In section methods, the CWCR and IWCR are described. And then, the performance evaluation indicator is introduced. In section results, a case study of TGHS in China is discussed. In Section conclusions, relevant conclusions are drawn.

\section{Methods}

Because the ECM method is mainly used in long term power generation scheduling, the RC method is not applicable when there are many units of HS. Therefore, the CWCR method is the basic method of this paper.

\subsection{Classical water consumption rate method (CWCR)}

The operation benefit of HS can be measured by three types of indicators, namely absolute dynamic indicators such as head, flow and input energy, unit dynamic indicators such as efficiency, WCR and differential dynamic indicators such as flow increment. Among these indicators, WCR is the main indicator to evaluate the operation efficiency of HS, which is calculated as follows

$$
R=\frac{Q}{N}
$$

Where, $Q$ represents the generation flow of the whole plant, and $N$ represents the output of the whole plant. $R$ is the WCR of the whole plant.

The most relevant indicator with WCR is gross head and net head. Therefore, scholars or actual dispatchers often fit the functional relationship between WCR and gross head based on historical operation data, namely

$$
R=f\left(H_{\text {gross }}\right)
$$

Where, $H_{\text {gross }}$ is gross head for the whole plant. For the HS with multiple units, there are vast differences in efficiency among different units, and the calculating method about head loss among units is different. Utilizing the net head of whole plant may lead to big deviation. Therefore, we select the gross head as the parameter of formula (2).

$$
H_{\text {gross }}=\left(Z_{0}+Z_{1}\right) / 2-Z_{\text {down }}
$$

Where, $Z_{0}$ is the initial upstream water level of $\mathrm{HS}, \mathrm{Z}_{1}$ is the final upstream water level, and $\mathrm{Z}_{\text {down }}$ is the tail water level.

When utilizing the formula (2) to conduct output calculation, the gross head should be obtained first. Then, the WCR curve is queried to forecast the WCR. Finally, the generation flow is calculated by output multiplied by WCR, namely

$$
Q=R * N
$$

\subsection{Improved WCR method considering aftereffects (IWCR)}

In long term power generation scheduling, the relationship between gross head and WCR is a one-toone and steady-state relationship. However, when the time scale of dispatching model is hour, the corresponding relation of formula (2) is dynamic and has aftereffects. This kind of aftereffects mainly comes from the aftereffect of the tail water level of HS. Based on this, we propose an IWCR method considering aftereffects, namely

$$
R_{t}=f\left(H_{\text {gross }}, R_{t-1}, R_{t-2}, \ldots, R_{t-\tau}\right)
$$

Where, $t$ represents the time period number. $R_{t}$ represents the WCR at $t$ th period. The value of $\tau$ needs to be determined by operation data of HS, representing aftereffects of WCR.

\subsection{Performance evaluation}

In this paper, we introduce the mean absolute deviation $(M A D)$ to evaluate the predictive performance of different WCR models. Meanwhile, we also make some statistics on the predicted WCR in different relative deviation intervals

$$
M A D=\frac{\sum_{t=1}^{T}\left|R_{t}^{o}-R_{t}^{s}\right|}{T}
$$

where $R_{t}^{o}$ means the observed WCR at the $t$ th period. $R_{t}^{s}$ means the simulated or forecasted WCR at the $t$ th period. The lower the value of $M A D$ is, the stronger the prediction accuracy of WCR has.

\section{Results}

\subsection{Research area and dataset}


Located in the main stream of the Yangtze River, China, the Three Gorges hydropower station (TGHS) is located in the main Stream of the Yangtze River. There are 34 water turbine generators in total, which belong to 8 types of units respectively. Considering the efficiency and accuracy of calculation, the Three Gorges Cascade Communication and Control Center (TGCRCCC) does not refer to the unit level when developing daily generation plan. Therefore, how to improve the accuracy of WCR of TGHS is a study of high practical value.

The study of this paper tries to improve the predictive accuracy of WCR with hour scale. Therefore, the operation data with 2-hour scale of TGHS in 2013-2015 is selected, including the upstream water level, the tail level, the output and generation flow of left and right bank. 16 units and 18 units are located in left and right bank respectively. Since, there are some differences in operation efficiency of left and right bank, so we divide the whole plant into left and right bank to improve the predictive accuracy.

\subsection{CWCR curve fitting}

In the actual dispatching process, the relationship between gross head and WCR is usually fitted by using data with daily scale. Figure 1 shows the scatterplot between gross head and WCR on daily scale of TGHS in 2013-2015:

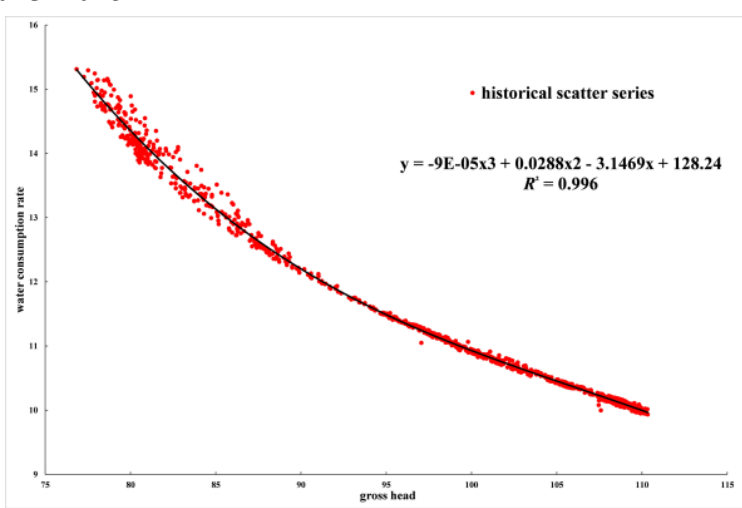

Fig. 1. The scatterplot between gross head and WCR of TGHS on daily scale.

The function relationship between gross head and WCR is fitted as follows:

$$
y=-0.00009 x^{3}+0.0288 x^{2}-3.1469 x+128.24
$$

Where, $y$ represents the WCR of the whole plant, and $x$ represents the gross head.

From the fitting effect, the cubic polynomial can characterize the relationship between gross head and WCR commendably, and the determination coefficient $R^{2}$ reaches 0.996 .

\subsection{Determination of input variables and comparative experiments}

From Formula (5), the value of $\tau$ needs to determined when considering aftereffects of WCR. Firstly, the WCR of the left and right bank of TGHS can be regarded as time series respectively, and the autocorrelation and partial autocorrelation of WCR in different periods are calculated. The 2-hour scale correlation results of the left and right bank of TGHS during 2013-2015 are shown in Figure 2-5.

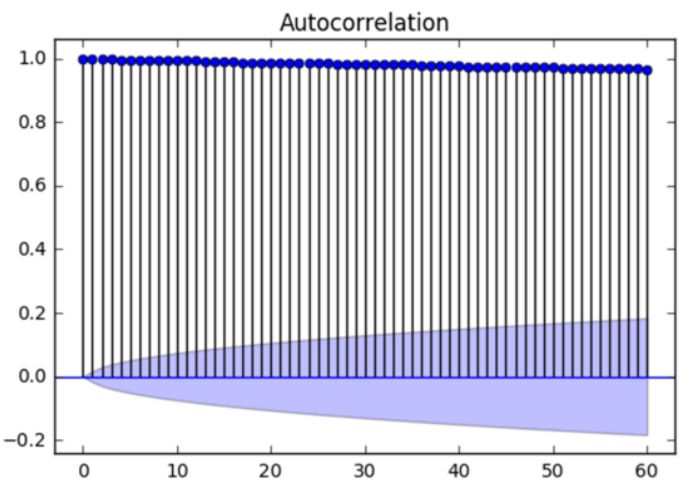

Fig. 2. The autocorrelation of left bank

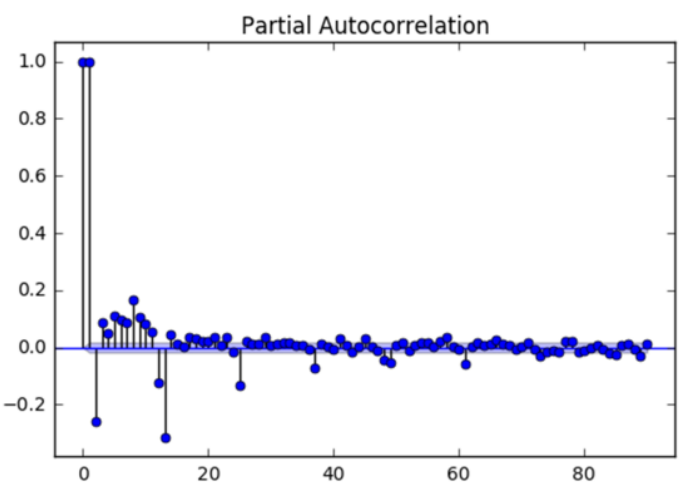

Fig. 3. The partial autocorrelation of left bank.

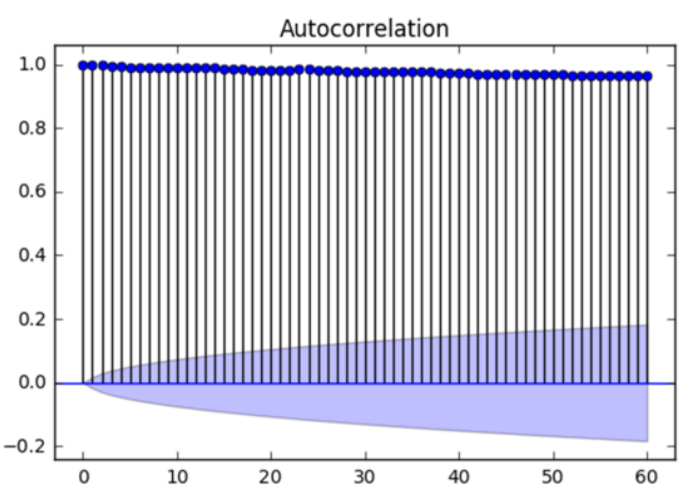

Fig. 4. The autocorrelation of right bank.

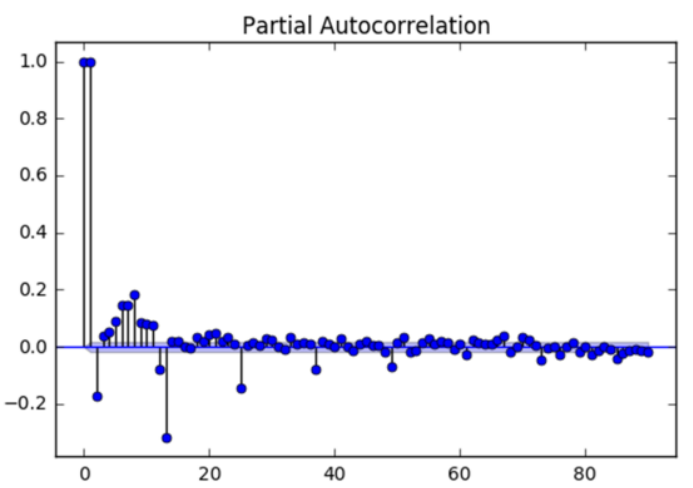

Fig. 5. The partial autocorrelation of right bank. 
To improve the prediction accuracy, artificial neural network (ANN) is used to fit the formula (5). Simultaneously, in Section CWCR curve fitting, we fit the CWCR curve between WCR of the whole plant and gross head. Therefore, the prediction results of WCR with CWCR and IWCR during 2016 of TGHS are listed in Table 1.

Table 1. The statistical indicators of WCR deviation with different prediction methods.

\begin{tabular}{|c|c|c|c|}
\hline Index & IWCR_L & IWCR_R & CWCR_W \\
\hline$M A E$ & 0.032 & 0.041 & 0.61 \\
\hline$[0,0.1]$ & 4049 & 3942 & 55 \\
\hline$(0.1,0.2]$ & 256 & 281 & 121 \\
\hline$(0.2,0.3]$ & 35 & 89 & 133 \\
\hline$(0.3,1]$ & 19 & 47 & 4060 \\
\hline Total_NO & 4359 & 4359 & 4369 \\
\hline
\end{tabular}

Note: IWCR L, IWCR R and CWCR W represent the left bank, the right bank and the whole plant of TGHS respectively. The unit of $M A E$ is $\mathrm{m}^{3} /(\mathrm{s} \cdot \mathrm{wkw})$. [0, 0.1$]$ represents that the relative deviation between observed and forecasted WCR of TGHS. Total_NO represents the total test periods in 2016 of different methods.

\section{Results}

Seen from Figure 2 and 4, the autocorrelation plots of two banks of TGHS all present an obvious trailing phenomenon, indicating that there are strong aftereffects about WCR. Autocorrelation characterizes the influence of all previous historical WCR on the current WCR. However, partial autocorrelation only considers the influence of $R_{t-\tau}$ on $R_{t}$. Seen from Figure 3 and 5, when the value of $\tau$ is equal to one, the partial autocorrelation is close to one. Therefore, the value of is determined to 1 , and the formula (5) can be rewritten as follows:

$$
R_{t}=f\left(H_{\text {gross }}, R_{t-1}\right)
$$

The data preprocessing needs to be checked before conducting the comparative experiment. We sum up the single output of 34 units of TGHS and gain the total output. Then the feasible period is selected if the deviation between the total output and the observed output of whole plant is less than $2 \mathrm{wkw}$. Therefore, the total test periods in IWCR and CWCR are different.

The accuracy of IWCR is significantly higher than that of CWCR, and the $M A E$ of left and right bank is decreased by $0.578 \mathrm{~m}^{3} /(\mathrm{s} \cdot \mathrm{wkw})$ and $0.569 \mathrm{~m}^{3} /(\mathrm{s} \cdot \mathrm{wkw})$ respectively compared with the $M A E$ of whole plant. Meanwhile, the IWCR model produces more high- precision predictions due to considering aftereffects of WCR. In the interval of relative deviation less than 0.1 , the periods of WCR of left and right bank increase 3994 and 3887 periods respectively compared with the period of WCR calculated by CWCR in this interval. Therefore, the IWCR model, which is equipped with aftereffects, can significantly improve the prediction accuracy of WCR.

Accurate WCR means that dispatchers will have a small deviation in estimating the power generation flow of TGHS from formula (4). The tailwater level is highly correlated with the power generation flow, so the tailwater level of TGHS will be more accurate. Furthermore, the inflow of Gezhouba hydropower station is basically determined by outflow of TGHS. Therefore, the estimating inflow will be more accurate, and the jacking effects of Gezhouba will be more reasonable to estimate. What's more, the risk of frequent adjustment of TGHS and Gezhouba cascade stations will also be lower. Therefore, accurate WCR can provide reliable basis for the output calculation of short-term and real-time generation dispatching of TGHS and Gezhouba.

\section{Conclusions}

For the problem of rapid and accurate output calculation in large HS with multiple units, an improved water consumption rate method considering aftereffects is proposed. A case study of TGHS is selected to compare the prediction results of IWCR with CWCR with 2-hour scale. Two conclusions can be drawn as follows: (1) The IWCR considering aftereffects of WCR significantly improves the prediction precision of WCR for TGHS compared to CWCR, and the MAE indicators of left and right bank reduce $0.578 \mathrm{~m}^{3} /(\mathrm{s} \cdot \mathrm{wkw})$ and 0.569 $\mathrm{m}^{3} /(\mathrm{s} \cdot \mathrm{wkw})$ respectively. (2) There are more prediction WCR periods distributed over low relative deviation interval. So, when the TGCRCCC estimates the output or generation flow, the IMCR can produce less calculation deviation, and the plan scheme is more consistent with the actual operation process. Therefore, the frequent adjustments on the operation scheme can be avoided, and the security the TGHS is also higher.

Besides, through statistics, in non-flood season (December and January- April), the IWCR method can significantly reduce the prediction deviation of WCR. But in the flood season (May-September), there still exist some periods with large WCR deviation of left and right bank of TGHS. Meanwhile, Seen from Figure 3 and 5 , the partial autocorrelation coefficients of two bank all show exponential decay when $\tau$ is equal to $13,25,37,49$. Therefore, how to further explore the law of WCR prediction is the direction that we mainly focus on in the future.

This work is supported by the National Key R\&D Program of China (No. 2016YFC0402205, No. 2016YFC0402210), and the National Natural Science Foundation Key Project of China (No. 91547208, No. U1865202). 


\section{References}

1. C. M. Ji, H. J. Yu, J. J. Wu, Water, 10, 808- (2018)

2. Z. Z. He, J. Z. Zhou, H. Qin, Eng. Appl. Artif. Intell. 81, 1-13 (2019)

3. X. H. Wen, J. Z. Zhou, Z. Z. He, C. Wang, Water, 10, 383-(2018)

4. L. M. F. Guilherme, C. F. Erlon, L. D. M. Vitor, Renew. Energy, 131, 45-54 (2019)

5. B. J. Jia, J. Z. Zhou, X. Chen, IEEE Access, 99 (2019)

6. J. Zhao, Jilin Water Resources (2014)

7. Y. Chen, G. W. Ma, D. H. Yang, Water Power (2009)

8. Z. Yang, K. Yang, L. Su, Math. Probl. Eng. PT.10, 1-29 (2018)

9. H. Hu, K. Yang, L. Lang, Water Resour. Manag. 33, 2357-2379 (2019)

10. Z. K. Feng, J. N. Wen, Z. Q. Jiang, Water Resour. Manag. 34, 2029-2041 (2020) 